\title{
AUTO-APRENDIZAGEM DIRIGIDA EM NATAÇÃO
}

Silmara Cristina Lazarini* Mauricio Tadeu Frajacomo**

\section{Resumo:}

Num país que possui grande quantidade de água, a necessidade da preservação da vida se faz presente pela segurança e lazer. Assim, o aprendizado da natação deve ocupar lugar de destaque nas atividades propostas. A exemplo de algumas décadas anteriores, onde não havia tantas escolas de aprendizagem de natação e os clubes onde a modalidade era praticada, a natação era destinada somente àqueles que pretendiam treinar para possivelmente se transformar em atletas, a aprendizagem restringia-se em sua grande parte na observação dos praticantes da modalidade. Nesse sentido, o presente estudo tem por objetivo analisar o tempo de duração de aprendizado na natação através da imitação dos movimentos, ou seja, da Auto Aprendizagem Dirigida. Através de filmagens e observações foi possível constatar que essa nova metodologia atingiu mais rapidamente seu objetivo no que se diz respeito aprender a natação para sobrevivência.

Palavras-chave: Natação Utilitária, Auto-Aprendizagem, Nova Metodologia.

\section{Introdução}

A natação é tão antiga quanto o homem e este teria aprendido a sustentar-se na água por instinto de sobrevivência e por observação dos animais (VELASCO, 1994).

Atualmente as pessoas procuram a natação para melhorar a saúde, pois as propriedades da água produzem efeitos benéficos, não só ao físico, mas também ao psíquico. Desta forma, nadar passou a ter um significado diferente, principalmente porque o Brasil é imensamente repleto de locais para divertimento em que a água

* Graduada em Bacharel e Licenciatura em Educação Física pelo Centro Universitário de Araraquara Uniara, Graduada em Pedagogia pela Faculdades de Pinhais - Fapi, graduanda do curso de Nutrição no Centro Universitário de Araraquara - Uniara e cursando pós-graduação Lato Sensu em Nutrição, Atividade Física e Qualidade de vida pelo Centro Universitário de Araraquara - Uniara.

** Mestre em Desenvolvimento Regional e Meio Ambiente pelo Centro Universitário de Araraquara Uniara, Especialista em Treinamento Desportivo pela Fundação Padre Albino, Graduado em Educação Física pela Fundação Educacional de São Carlos e em Pedagogia pela Faculdades de Pinhais - Fapi. 
está presente, assim a aprendizagem da natação não provém apenas da melhora da qualidade de vida, mas também por sobrevivência.

Com o aumento do interesse das pessoas pela natação, houve também um aumento significativo de escolas desta modalidade o que obrigou os profissionais desta área a buscarem melhorias no ensino da mesma (MACHADO, 1978).

O processo de aprendizagem pode ser definido como uma mudança interna do indivíduo, ocasionando uma melhora relativa e permanente em seu desempenho, como resultados da prática. A educação do indivíduo deve ser analisada como um todo, ou seja, o bom entendimento dos domínios cognitivos (intelectual), afetivo (emoções) e motor (movimento) podem servir como base para garantir o processo de ensino-aprendizagem de forma que envolva o indivíduo por inteiro.

Os domínios do comportamento configuram o processo de aprendizagem em três fases:

A) Primeira infância: corresponde aos dois primeiros anos de vida e o sujeito ainda está completamente imaturo para o desenvolvimento de habilidades, porém com um grau de atenção muito alto.

B) Segunda infância: corresponde do terceiro ao sexto ano de vida e o principal objetivo é oferecer uma vasta experiência nas diversas modalidades esportivas para que se crie o hábito da pratica de atividades físicas.

C) Terceira infância: compreende dos sete aos doze anos de idade e é chamada de "fase de ouro" (facilidade de aprendizado), os domínios do comportamento devem ser trabalhados harmonicamente e o erro deve ser entendido como fator natural e auxiliar da aprendizagem.

Considerando que a terceira infância é a melhor fase para o aprendizado de uma habilidade, os movimentos tornam-se se uma ferramenta na variedade de atividades motoras complexas da vida diária (MAGILL, 1998).

Tani (1988) afirma que a criança no período de sete a doze anos deve refinar os movimentos fundamentais, aperfeiçoando-os e combinando-os entre si, proporcionando a diversificação e, como conseqüência, o aumento significativo de experiência motora. Essa evolução deve seguir uma ordem crescente de complexidade, entendido como aumento na interação entre os elementos do comportamento. O padrão de comportamento é adquirido e posteriormente diversificado, ou seja, realizado de forma variada em termos de direção, velocidade, força, dentre outros parâmetros. Após vários padrões adquiridos, esses interagem para formar novos padrões.

Machado (1978) ressalta que ensinar a nadar está intimamente ligado pela simples palavra ensinar. Diz ainda que o ensino da natação sofreu a ação de muitas correntes, mas que através do tempo, se aperfeiçoou até ser capaz de satisfazer as necessidades

REVISTA UNIARA, $n .^{0} 21 / 22,2008 / 2009$ dos alunos. Ele ainda relata que a execução dos gestos aprendidos era feita por etapas e repetidos até que o todo fosse assimilado e compreendido.

Sabemos que as crianças aprendem melhor brincando e se divertindo e que o processo de aprendizagem é desencadeado por fatos novos. Tudo o que atrai a atenção das crianças lhe permite tentar experimentar.

Dessa forma nota-se que as mudanças metodológicas são necessárias para melhorar cada vez mais o ensino da natação. É evidente que toda prática metodológica está intrinsecamente ligada à filosofia de trabalho de cada um, porém a atenção das aulas deve estar voltada apenas aos alunos, procurando agradá-los de forma a deixar a aula mais excitante e ao mesmo tempo sem perder as riscas metodológicas adotadas.

A pretensão deste estudo foi introduzir uma nova metodologia que ajude os profissionais da área a ensinarem a natação utilitária, istoé, a natação de sobrevivência, com maior motivação para os alunos e com alcance mais rápido de resultados, favorecendo tanto aos sujeitos praticantes da modalidade quanto ao professor que poderá introduzir novos exercícios de acordo com o desempenho dos alunos. Essa nova metodologia permite que os indivíduos realizem os movimentos observados com maior liberdade de expressão e sem depender rigorosamente do professor para realizar as atividades exigidas, ou seja, através da observação de alguns gestos realizados por sujeitos que conheçam e dominem o movimento, os indivíduos são capazes de imitar rudimentarmente os gestos e realizá-los de forma que o educador apenas oriente certas correções para facilitar a execução do mesmo. Nesse caso, o processo de Auto Aprendizagem se baseia apenas no ensino da sobrevivência, ou em cursos "relâmpagos", cursos de férias oferecidos em clubes, onde a demanda de sujeitos é muito grande e o professor é incapaz de promover um ensino personalizado.

Desta forma, aplicando-se a Auto Aprendizagem Dirigida, os indivíduos aprenderão a natação rudimentar num tempo bem pequeno, caso haja interesse na continuação do aprendizado dos estilos, então o professor poderá utilizar a metodologia tradicional, pois o papel do professor é essencial para o aprendizado completo da natação.

\section{Materiais e métodos}

Para essa pesquisa foram selecionadas 48 crianças no estágio da "fase de ouro" do desenvolvimento motor de 7 a 12 anos, de ambos os sexos. Para a seleção dos sujeitos foi adotado o critério de "não saber nadar", para que todos fossem iniciantes. Os sujeitos foram divididos em 4 grupos mistos de 12 alunos. Foram determinados os grupos Ae B para aqueles que aprenderiam a natação pelo método tradicional, denominado "grupo Controle", na qual os indivíduos executariam os movimentos passo a passo, e os grupos $\mathrm{C}$ e $\mathrm{D}$ com sujeitos que aprenderiam pela Auto 
Aprendizagem Dirigida, ou seja, deslocando-se na água através da imitação/ reprodução dos movimentos observados.

Cada grupo teve um professor diferente para que não fosse analisada a influência do profissional no processo de aprendizagem, mas sim a duração da mesma.

Para a obtenção dos resultados foi utilizado uma Câmera Panasonic Palmcorder IQ modelo $\mathrm{n}^{\circ}$ PV-D406 D, DC 6V, 8.1W (11.1 W Max) digital EIS 22x /16x optical zoom Hi Tech Head, juntamente com um diário de anotações, na qual foram redigidas todas as informações sobre as aulas dos quatros grupos e de cada sujeito.

A pesquisa aconteceu num período de 8 meses com aulas 2 vezes na semana, com duração de 45 minutos, em piscina aquecida e com altura apropriada para a faixa etária pesquisada. Os critérios utilizados para a mensuração dos resultados foram: a) imersão com duração de até 10 segundos, b) flutuação durante 15 segundos aproximadamente em decúbito ventral, c) propulsão de pernas em 15 metros, d) propulsão de braços em 15 metros. e) coordenação dos movimentos com respiração lateral em 25 metros. A pesquisa foi colocada em prática após ter sido aprovada pelo comitê de ética da Uniara.

Os grupos controle (A e B) iniciaram suas aulas seguindo os critérios adotados e com os seguintes exercícios:

A) imersão: exercícios em apnéia e educativos para o aprendizado da respiração.

B) flutuação: o auxilio do professor foi importante até que os alunos sentissem segurança para realizarem sozinhos o exercício.

C) propulsão de pernas: exercícios com pranchas e auxilio do professor até a realização autônoma .

D) propulsão de braços: exercícios na prancha e auxilio do professor até a realização autônoma.

E) coordenação dos movimentos com respiração lateral: exercícios com auxilio do professor individualmente, com a prancha e o nado completo.

O grupo da Auto Aprendizagem Dirigida (C e D) seguiu os mesmos critérios apresentados da seguinte forma:

- Antes de iniciar os critérios as crianças foram submetidas a brincadeiras dentro da piscina para uma melhor adaptação ao meio.

- Após a adaptação, os critérios foram um por um demonstrados pelo professor a fim de que os alunos imitassem de acordo com a sequiência proposta.

- Seguido à demonstração e imitação de cada critério foram realizadas atividades lúdicas para a automatização dos gestos observados.

\section{Resultados e discussão}

Os resultados foram coletados para cada critério, analisando o desempenho

REVISTA UNIARA, $n .^{0} 21 / 22,2008 / 2009$ individual de cada grupo.

$\mathrm{Na}$ amostra da imersão em até 10 segundos, a porcentagem de indivíduos que conseguiram realizar o exercício foi: $\mathrm{A}=20 \%, \mathrm{~B}=24 \%, \mathrm{C}=28 \%$ e $\mathrm{D}=28 \%$. Assim, os alunos da Auto Aprendizagem Dirigida $(\mathrm{CeD})$ demonstraram melhores resultados, pois dos 12 estudados, 8 , respectivamente alcançaram o objetivo proposto, enquanto que os alunos do grupo controle, do grupo A, somente 6 conseguiram $\mathrm{e}$ do grupo B, 7. Diante disso, a pesquisa nos revela que esse fato de copiar os gestos facilita a aprendizagem dos alunos.

No critério da flutuação em decúbito dorsal em 15 segundos, a porcentagem de indivíduos foi: $\mathrm{A}=22 \%, \mathrm{~B}=19 \%, \mathrm{C}=27 \%$ e $\mathrm{D}=32 \%$. Foi possível analisar que o grupo $\mathrm{D}$ atingiu melhor o objetivo. Dos 12 sujeitos analisados, todos conseguiram realizar o exercício, juntamente com o grupo $\mathrm{C}$ que dos 12 sujeitos, 10 tiveram resultados satisfatórios. Nos grupos controle (A e B), tiveram respectivamente 8 e 7 sujeitos capazes de realizar o exercício. Novamente foi notório o melhor desempenho da metodologia proposta. De acordo com MACHADO (2003), cada trabalho se prende ao desafio de cópia de que o aluno conhece ou está vendo. Dessa forma, levamo-lo a conduzir-se na água como algo que conhece ou vê, mas segundo suas próprias possibilidades.

No terceiro critério, propulsão de pernas em 15 metros, o percentual de indivíduos em relação ao desempenho foi: $\mathrm{A}=24 \%, \mathrm{~B}=21 \%, \mathrm{C}=26 \%$ e $\mathrm{D}=29 \%$, onde os grupos $\mathrm{C}$ e $\mathrm{D}$ também obtiveram melhores resultados em relação aos grupos $\mathrm{Ae} \mathrm{B}$, ou seja, os grupos da metodologia da Auto Aprendizagem apresentaram respectivamente 10 e 11 sujeitos capazes de realizar o exercício, enquanto que os grupos da metodologia tradicional atingiram 9e 8. Segundo LIMA (1999), há alguns anos atrás, quando não havia processos pedagógicos mais elaborados, crianças que aprendiam a nadar nos rios e lagos realizavam movimentos mais naturais do que muitos alunos apresentam nas escolas de natação atualmente, pois eram movimentos livres, espontâneos.

No quarto critério, a porcentagem de indivíduos para propulsão de pernas em 15 metros foi: $\mathrm{A}=21 \%, \mathrm{~B}=18 \%, \mathrm{C}=25 \%$ e $\mathrm{D}=36 \%$, de forma que os sujeitos dos grupos A e B apresentaram respectivamente 6 e 5 aptos a realizarem o exercício. Neste critério a análise demonstrou certa defasagem no emprego dessa metodologia se comparado com os grupos C e D que, respectivamente, demonstraram ser superiores, atingindo 7 e 10 sujeitos capazes de realizarem o exercício proposto. De acordo com MAGILL (1984) o aprendizado da habilidade se torna fraco diante de repetições exacerbadas de um mesmo movimento, fazendo com que o indivíduo desanime do processo de aprendizagem. A preocupação conteudística da Auto Aprendizagem, preconiza esse fato, a partir de que o aluno 
tendo a liberdade de nadar de acordo com suas próprias possibilidades ele tem a liberdade de criar mecanismos que o facilitem ou até mesmo o dificultem na execução de exercícios.

No último critério, coordenação dos movimentos com respiração lateral em 25 metros a porcentagem de indivíduos aptos foi: $\mathrm{A}=24 \%, \mathrm{~B}=21 \%, \mathrm{C}=29 \%$ e $\mathrm{D}=$ $26 \%$. A análise final mostrou que os grupos $\mathrm{Ce} D$ atingiram respectivamente 10 e 11 sujeitos capazes de se deslocarem em 25 metros sustentando o nado crawl, enquanto que os grupos A e B atingiram 9 e 8 . Portanto, neste critério que é considerado padrão para a análise da metodologia proposta, aAuto Aprendizagem Dirigida obteve melhores resultados. Araujo Jr. (1993, p.29) afirma que a natação tinha caráter utilitário, de segurança e de sobrevivência. Não importava se os braços eram alternados ou simultâneos, se a cabeça estava alta ou baixa. $\mathrm{O}$ autor completa dizendo que os índios nadavam como sabiam, como seus antepassados the transmitiram,desprendidos de conceitos preestabelecidos, de técnicas de execução, de sofisticados locais.

Em aproximadamente 68 aulas ( 8 meses de pesquisa já considerando imprevistos e feriados) foi possível constatar que na análise do número de aulas necessárias para que os sujeitos aprendessem a nadar foi significativamente maior na nova proposta de metodologia, isto é, respectivamente os grupos $\mathrm{C} \mathrm{e} \mathrm{D}$ alcançaram seus objetivos em 16 e 13 aulas, em contra partida os grupos Ae B levaram 18 e 19 aulas. Dessa forma, Machado (2003) relata em seu livro que a Auto Aprendizagem Dirigida é um método alternativo. É a cópia do gesto como forma de aprendizado. Apesar de parecer paradoxo, utiliza a aprendizagem descoberta pelo próprio aluno, mas sob direção e supervisão do professor.

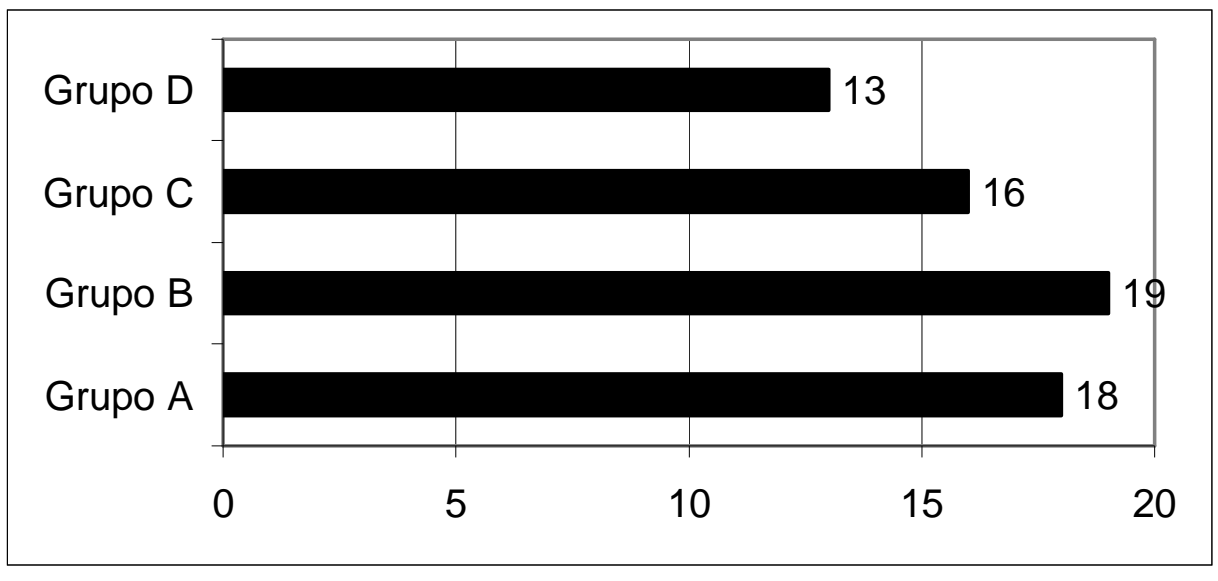

Quanto ao desempenho final, no tocante ao tempo necessário para aprender a nadar, a Auto Aprendizagem Dirigida entra para a literatura comprovando um novo método capaz de melhorar o tempo de aprendizagem dos sujeitos e principalmente fazendo com que o professor possa avançar mais rápido com o ensino dos outros estilos e outras atividades, de acordo com o objetivo proposto, ou seja, na média dos grupos A e B encontramos um total de 42,84 aulas para que os sujeitos pudessem aprender a nadar, em contra partida, a média dos grupos $\mathrm{C}$ e D foi de 25,16 aulas para que os alunos atingissem o objetivo.

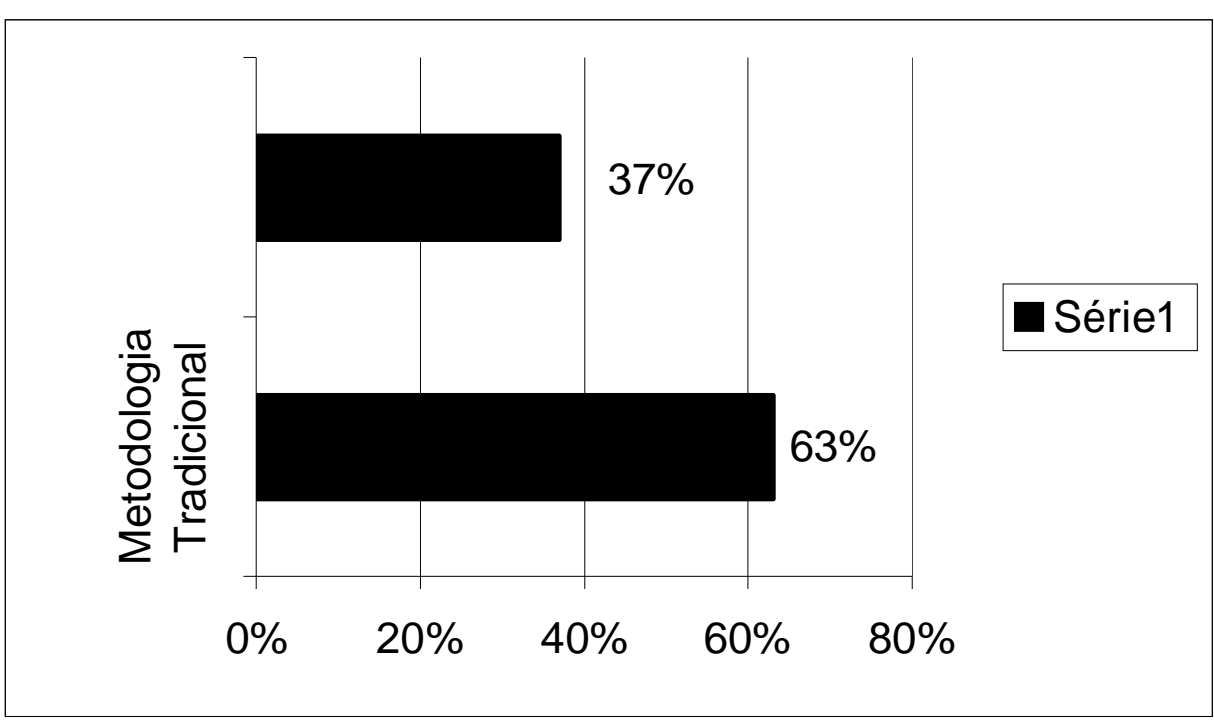

\section{Considerações finais}

Portanto, a pesquisa expõe que a Auto Aprendizagem Dirigida é um novo método que pode ser adotado pelos profissionais da área a fim de facilitar o processo ensino-aprendizagem na natação utilitária, ou seja, os sujeitos se tornam mais livre no processo de aprendizagem e o professor têm a possibilidade de avançar com seu conteúdo programático mais rápido. Os resultados dessa pesquisa comprovam que para a natação utilitária, a Auto Aprendizagem é o método mais indicado em detrimento a duração de aprendizagem, pois o professor ministrando um curso de férias (30 dias), consegue, através desse método, ensinar a natação utilitária até o fim do curso, proporcionando aos sujeitos a sobrevivência na água e o nado rudimentar do crawl e reforça a teoria que a observação tem um papel indispensável no processo de resposta ao que se pretende ensinar. Portanto, foi possível verificar que a Auto Aprendizagem Dirigida, mesmo pulando algumas etapas fundamentais da confiança do aluno na fase de adaptação, na exploração dos métodos de sustentação e informação das etapas posteriores é
REVISTA UNIARA, $n .^{0}$ 21/22, 2008/2009 
o melhor método para que a criança aprenda ao menos para a sobrevivência.

\section{Referências bibliográficas:}

ARAUJO Jr.B. Natação: saber fazer ou fazer sabendo? Campinas: Editora da Unicamp, 1993.

CORRÊA, Célia Regina Fernandes; MASSAUD, Marcelo Garcia. Escola de natação: montagem, administração, organização pedagógica, do bebê à competição. Rio de Janeiro: Sprint, 1999.

CORRÊA, Cleia Regina Fernandes; MASSAUD, Marcelo Garcia. Natação na idade escolar: terceira infância: a natação no apoio ao aprendizado escolar. Rio de Janeiro: Sprint, 2004.

DAMASCENO, Leonardo G. Natação para bebês dos conceitos fundamentais à prática sistematizada, Revista Brasileira de Educação Física, v.1, p.78-97, 2001.

GAMBRIL, Doanald D. Natação moderna. Rio de Janeiro: Fórum, 1969.

KERBEJ, Francisco Carlos. Natação algo mais que 4 nados. São Paulo: Manole, 2002.

LEAL, Clésio Vargas. Profissional de natação: 100 estratégias para o sucesso. Rio de Janeiro: Sprint, 2003.

LIMA, Willian de. Ensinando natação. São Paulo: Phorte Editora, 1999.

MACHADO, David Camargo; Metodologia da natação. 2. ed. São Paulo: Editora EPU, 2003.

Metodologia da natação. São Paulo: Editora EPU, 1978.

MAGILL, Richard A; Aprendizagem motora: conceitos e aplicações. 5. ed. São Paulo: Edgard Blucher; 1984.

MAGILL, Richard A. Aprendizagem motora. 9. ed., São Paulo: Edgard

REVISTA UNIARA, $n .^{\circ} 21 / 22,2008 / 2009$
Blucher, 1998.

MAKARENKO, Leonid P. Natação: seleção de talentos e iniciação esportiva. Edson de Godoy Palomares (Trad.). Porto Alegre: Artmed Editora; 2001.

MANUAL do treinador de natação; nível trainee. Belo Horizonte: Editora FAM, 1999.

MASSAUD, Marcelo Garcia. Natação 4 nados: aprendizado e aprimoramento. Rio de Janeiro: Sprint, 2001.

PESSOA FILHO, D.M.; KOKUBUN, E. Força e potencia são determinantes do desempenho em velocidade máxima e sub-maxima de nados entre nadadores habilidosos. In: CONGRESSO BRASILEIRO DE BIOMECÂNICA, 7. Florianópolis. Anais... Florianópolis: Universidade Federal do Rio Grande do Sul, 1999. p.113-118.

REBELATTO, J.R.; CONCEIÇÃO, J.G; Natação para bebês: considerações educacionais e fisiológicas. Revista Brasileira de Ciência e do Esporte, v.10, n.2, p. 60-4; 1989. Disponível em: <www.lilacssp.com.br>. Acesso em: 12 jun. 2006.

TANI, G.; Educação Física escolar: fundamentos de uma abordagem desenvolvimentista; São Paulo; EPU-Edusp, 1989.

TANI, G.; Significado, detecção e correção do erro de performance no processo ensino-aprendizagem de habilidades motoras. Revista Brasielira de Ciência e Movimento, v.3, p.50-58, 1989.

UGRINOWITSCH, H.; et al; Freqüência de feedback como um fator de incerteza no processo adaptativo em aprendizagem motora. Revista Brasileira de Ciência e Movimento. v.11, n.2, p.41-47, 2003.

VELASCO, C.G. Natação segundo a psicomotricidade. 2. ed. Rio de Janeiro: Sprint; 1997. 


\section{Title:}

\section{SELF-DIRECTED LEARNING IN SWIMMING}

\section{Abstract:}

In a country that has plenty of water it is necessary to preserve life for security and leisure reasons. Therefore, learning how to swim must occupy a central place in the proposed activities. In some previous decades, there were not many swimming schools and in the clubs where the modality was practiced, swimming was only destined to people who intended to practice swimming in order to possibly become athletes. Most of time learning was based on observing sportspeople who practiced the modality. Thus, this study aims at analyzing the swimming learning length by means of imitating movements, that is, the self-directed learning. By filming and observations we were able to verify that this new methodology reached its objective faster in what concerns swimming learning for survival.

Keywords: Utility Swimming, Self-Learning, New Methodology. 